\title{
Using Various Types of Multimedia Resources to Train System for Automatic Transcription of Czech Historical Oral Archives
}

\author{
Josef Chaloupka, Jan Nouza, and Michaela Kucharova \\ Institute of Information Technology and Electronics, Technical University of Liberec \\ Studentska 2, 46117 Liberec, Czech Republic \\ josef . chaloupka@tul.cz \\ https://www.ite.tul.cz/itee/
}

\begin{abstract}
Historical spoken documents represent a unique segment of national cultural heritage. In order to disclose the large Czech Radio audio archive to research community and to public, we have been developing a system whose aim is to transcribe automatically the archive files, index them and make them searchable. The transcription of contemporary ( 1 or 2 decades old) documents is based on the lexicon and statistical language model (LM) built from a large amount of recent texts available in electronic form. From the older periods (before 1990), however, digital texts do not exist. Therefore, we needed a) to find resources that represent language of those times, b) to convert them from their original form to text, c) to utilize this text for creating epoch specific lexicons and LMs, and eventually, d) to apply them in the developed speech recognition system. In our case, the main resources included: scanned historical newspapers, shorthand notes from the national parliament and subtitles from retro TV programs. When converted into text, they allowed us to built a more appropriate lexicon and to produce a preliminary version of the transcriptions. These were reused for unsupervised retraining of the final LM. In this way, we significantly improved the accuracy of the automatically transcribed radio news broadcast in 1969-1989 era, from initial $83 \%$ to $88 \%$.
\end{abstract}

Keywords: historical audio archives, speech-to-text transcription, OCR, lexicon building, machine learning.

\section{Introduction}

Historical spoken documents represent a unique segment of national cultural heritage. They authentically illustrate events, ideas, language, personalities and atmosphere of the past and present. Usually they have form of audio (or audiovideo) files originally recorded and stored on various types of fragile carriers. Therefore, for many years, their content was available only to a limited number of people. Due to the recent progress in information processing technology, namely digitization, speech-to-text transcription software and Internet, they can

A. Petrosino, L. Maddalena, P. Pala (Eds.): ICIAP 2013 Workshops, LNCS 8158, pp. 228-237, 2013.

(C) Springer-Verlag Berlin Heidelberg 2013 
be disclosed to any interested researcher or even to public. Let us mention at least several examples of oral archives that have been made accessible, recently: the US National Gallery of the Spoken Word [1, the Dutch collection of historical oral records [2], audio-video library HistoryMakers [3], or international project MALACH [4. The process of recording, preserving, processing, tagging, indexing and searching speech-based information has been described from several points of view in 5 .

In 2011 we launched a large 4-year project supported by the Czech Ministry of Culture, whose aim is to disclose more than 100,000 spoken documents collected by the Czech Radio (and its predecessor Czechoslovak Radio) during its 90 years of broadcasting [6. For this purpose, we have adapted our previously developed large-vocabulary continuous speech recognition (LVCSR) system to deal with broadcast recordings in Czech and Slovak and designed modules for speech indexation and search. During the first 18 months of the project, we have processed about 75,000 audio files (with total duration of 30,000 hours) and created a demo version of the web service that allows for smart search in the transcribed data [7.

The large amount of the data processed within the first two years was possible due to one main reason: So far, we have focused on the transcription of the files from the last two decades (1990 to present). Within that epoch, the Czech Radio archive covers hundreds of different broadcast programs, including main evening news, political debates, discussions, talk-shows, regional news, daily commentaries or read feuilletons. All are provided in good audio quality and their automatic transcription can benefit from the lexicon and statistical language model (LM) developed for contemporary Czech. However, when moving deeper to the history, we face two additional challenges: a lower signal quality and a language that differs from the recent one. The latter aspect is related to the fact that former Czechoslovakia belonged to the Soviet block (till 1989) where all official media was controlled by a strong communist regime. Most archive documents from that era are news and reports highly influenced by propaganda. Yet, these audio files reflect one important epoch in national history and their disclosure helps younger generations to understand it better.

The main difficulty with adapting the LVCSR system to the language of previous epochs consists in the fact that there are no texts available in digital form from those older periods. This complicates the creation of the epoch specific lexicons and LMs, which are necessary for the reliable performance of the transcription system. In this paper, we show our solution to the problem. We try to utilize less common resources, like scanned and digitized newspapers, subtitles from retro TV programs or shorthand parliament notes. When converted into electronic form, pre-processed and analyzed, these are a good source of epoch specific names, words and phrases. We describe the procedures used to build a representative corpus of historical texts, and to employ it for lexicon and LM adaptation. In experimental section, we show that this approach yields a significant improvement in the transcription accuracy. 


\section{Spoken Archive Transcription System and Its Linguistic Module}

The speech transcription system is the most complex part of the Archive Processing and Accessing Platform (APAP). It converts audio recordings into text and provides detailed information on each processed document, each individual utterance (namely about speaker and language) and each word (including its pronunciation and exact time of occurrence). All these pieces of information are stored in a large database, they are indexed and made searchable. More technical details about the APAP can be found in [8]. In this contribution, we will focus mainly on its linguistic module.

The linguistic module consists of a lexicon (words with their pronunciations) and a statistical language model. Its version applicable for contemporary Czech has been based on a huge (11 GB) corpus of electronic texts, mainly newspapers published since 1990 to present. (This corpus will be referred to as CPaper.) A small subset of the corpus $(2 \mathrm{~GB})$ contains human made transcriptions of real speech, namely speech in selected major TV and radio programs broadcast during the last decade. (Let us denote it CBroad.) Recently, the size of the whole text corpus is $13 \mathrm{~GB}$ of data, and every year it increases by approx. 0.3 GB.

The corpus is regularly analyzed to identify the most frequent words that should be included in the lexicon. Its current version contains $551 \mathrm{~K}$ lexemes (words, word-forms and multi-word expressions) that occurred at least 10 times in the corpus. In spite of the lexicon's large size, the Out-of-Vocabulary (OOV) rate is still about $1 \%$, mainly due to the fact that Czech belongs to the languages with a very high degree of inflection. The corpus also serves as a source data for computing the statistical (N-gram based) LM. In our system, we use a bigram LM, which is a compromise between memory usage, computation speed and performance accuracy. Again, more details can be found in [8].

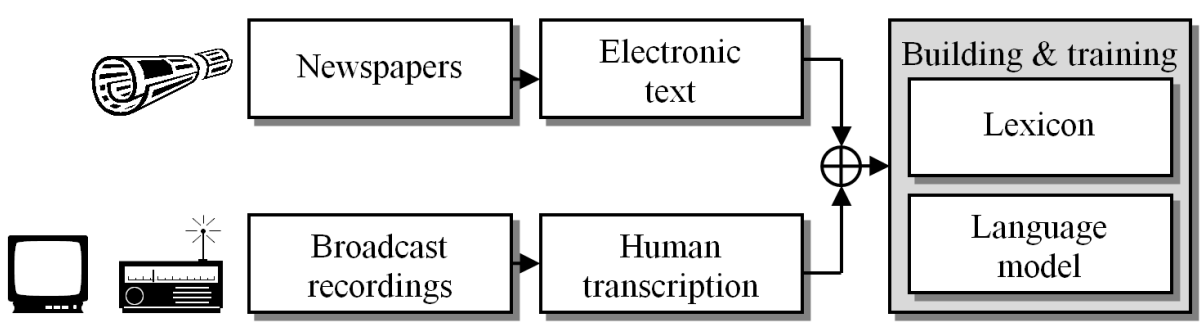

Fig. 1. Main resources used for building lexicon, and training statistical language model for speech recognition of contemporary Czech 


\section{Language Resources for Historical Document Transcription}

As explained in Introduction, year 1990 makes an important milestone in the audio archive. Before 1990, the language of media was much influenced by the communist regime and propaganda. The crucial question is how to adapt the transcription system to the speech of that historical epoch, i.e. how to teach the system the words, phrases, names and language structure that are not covered by the above mentioned corpus. The only effective solution consists in building a similar corpus that will represent the language before 1990. Because almost no electronic texts from that period exist, they must be acquired first. So far, we have discovered three major sources: old newspapers (in paper form), shorthand notes from parliament sessions and audio-visual retro programs with subtitles. Each source and its specific way of processing is described below.

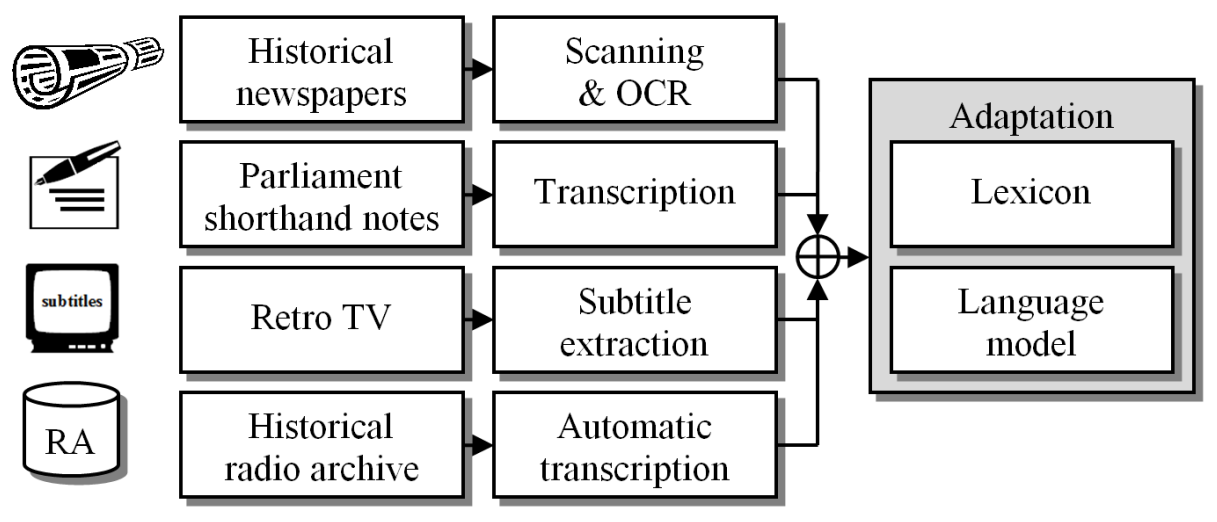

Fig. 2. Resources and procedures used to adapt the lexicon and language model to the period before 1990

\subsection{Historical Newspapers and Their Processing}

In the period of the communist regime (1948-1989), the main and the largest Czech newspaper was Rude pravo. It was the official press media of the Communist party. Recently, all its issues (with approx. 80 thousand pages) have been scanned by the Czech National Library. The same institution launched a project of their conversion (via an OCR software) into electronic texts. Up to now, years 1948 to 1983 have been processed. The OCR-based word error rates differ from page to page (typically in range 5 to $20 \%$ ), depending on the quality of the original copies. Unfortunately, many common methods used for OCR error reduction, like those based on statistical language modeling, could not been applied. It is logical, because a language model corresponding to that historical period does not exist, yet, and the application of the contemporary one would introduce irreversible loss in historical texts (e.g. changes in names or in spelling, replacements of epoch specific - and now obsolete - words by the modern ones, etc.) 
To convert the raw OCR output to the text suitable for our purpose, we have performed the following steps:

Page Filtering. For each page we measured the number of OOV strings. If the OOV rate was larger than $15 \%$ (the threshold found by the histogram analysis of the development data), the page was removed from the corpus, because either the original print or the scan were of very low quality.

Advertisement Blocking. To avoid repeated passages irrelevant for further linguistic analysis (namely advertisement, programs of TV, radio and theaters, etc) we have trained a simple key-word based classifier that identifies this type of unwanted text and removes it, too.

Safe Single-Letter-Error Correction. Using a small subset of OCRed texts we have detected the most frequent letter confusions (e.g. 'í' instead of 'i', 'l' instead of 'i', 'v' instead of 'y', 'é' instead of 'ě', etc). After that, we have compiled a list of the most frequent OOV strings that can be automatically and safely corrected. It was those strings that differed only in a single letter (one from the confusion pair list) from existing words in the contemporary lexicon. The correction was performed only if the considered string could be replaced by a unique lexicon item. In this way, the original number of 8.5 million OOV strings has been reduced to 6.4 million. The size of the newspaper texts is really large - $843 \mathrm{MB}$ (see Table 1). However, the last year covered in the corpus is 1983. Therefore, we had to search for another source that would represent the remaining years of the historic epoch (1984 to 1989).

\subsection{Historical Parliament Shorthand Records}

On the Czech Parliament web 9] we have found official shorthand records from all sessions of the Czechoslovak Federal Parliament and the Czech National Council held during their entire existence. We have chosen the latter institution because its documents contain only Czech, while in the Federal Parliament, both Czech and Slovak occurred. The records written originally by stenographers and now available in electronic form describe 93 sessions within the 1969-1989 period and include verbatim transcriptions of all speeches. To get a text resource appropriate for our purpose, we wrote a script that extracted only the transcriptions of verbal communication and removed the description of formal acts, like session opening and management, voting protocols, etc. In spite of its relatively small size, the acquired text offers at least some coverage of names and major events from the period 1984 to 1989.

\subsection{Subtitles from Retro TV Programs}

Since 1990s Czech TV has been producing a retro document series named 25 years ago. It is aired every day and contains a 5-minute-long selection of the most relevant news that were shown in TV exactly a quarter of century ago. In accord with the new public broadcast regulations, this type of program must be accompanied by subtitles. They can be downloaded from the Czech TV web site and used (with minimum additional effort) as another resource for the lexicon 
and LM adaptation. Their main advantage consists in the fact that they represent authentic broadcast speech in all its forms (read, planned and spontaneous) in the similar way as it occurred in the radio archive in that period.

\section{Adapting Linguistic Module for 1969-1989 Period}

One of the most interesting historical epochs in the radio archive is the 1969-1989 era. From this period, the archive offers a continual series of main (evening) news broadcast for 20 years. This is a unique collection of 7660 recordings (approx. 3800 hours of speech), valuable both from the historic as well as linguistic points of view. Its transcription and disclosure has been declared one of the top goals of the project and therefore we aimed at making a proper lexicon and language model fitted specially for this epoch.

Table 1. Statistics of text resources used for adaptation of lexicon and LM for 19691989 era

\begin{tabular}{|l||c|c|c|c|}
\hline Resource & $\begin{array}{c}\text { Covered } \\
\text { period }\end{array}$ & $\begin{array}{c}\text { Size } \\
{[\mathbf{M B}]}\end{array}$ & Size in words & $\begin{array}{c}\text { Selected } \\
\text { OOV words }\end{array}$ \\
\hline \hline Historical newspapers $(R P)$ & $1969-1983$ & 942 & $132,772,115$ & 5886 \\
\hline Parliament steno-notes (Parl) & $1969-1989$ & 23 & $3,359,474$ & 2773 \\
\hline Retro TV (Retro) & $1986-1988$ & 5 & 626,767 & 1261 \\
\hline Transcribed radio archive $(R A)$ & $1969-1989$ & 182 & $26,330,195$ & - \\
\hline
\end{tabular}

\subsection{Adapting Lexicon}

We took the pre-processed text data from the three sources described in section 3 and limited them to the 1969-1989 period. For each source, we compiled a frequency ordered list of those OOV strings that were not in the existing $551 \mathrm{~K}$ lexicon. In case of the scanned newspapers, the list contained 1,779,756 strings, from which more than $90 \%$ were error strings caused mainly by OCR. Even if we focused only on the terms with frequency higher than 20, we had to check more than 50,000 strings and manually pick up 5886 word candidates for addition to the lexicon. This tedious work could not be automated as most of the candidates were names of people, companies or institutions closely related to the given historical era. We often used Czech Wikipedia to verify the existence of these entities and their correct spelling. As to the other two sources, their processing was much easier, because they contained minimum typing or spelling errors. Here, we picked up the words with minimum frequency 2. Table 1 gives an overview of the relevant statistics on the three resources. The last column shows the numbers of lexicon candidates selected from each source. Because the three candidate lists had some overlap, the total number of added words was 7368 . The meaning of the last row will be explained later. 


\subsection{Adapting Language Model}

To compute an N-gram language model (a bigram LM in our case), we have to chose an appropriate text corpus and compute occurrence counts for all N-word sequences. Usually, a word which is not present in the corpus is removed from the lexicon. The sequences not seen in the corpus are assigned small probability values according to the applied smoothing method. In our system we prefer Knesser-Ney technique [10].

We have prepared and evaluated four different LMs based on the following corpora: a) $R P$ only, b) $R P+$ Parl + Retro, c) $C$ Broad $+R P+$ Parl + Retro and d) $C P a p e r+C B r o a d+R P+P a r l+R e t r o$. The first two language models were computed entirely from the historical data, the remaining ones employed the contemporary texts, too. Their parameters and the results achieved with them are presented in Table 2 .

\subsection{Unsupervised LM Adaptation}

In experiments described in section 5, we show that the transcription accuracy measured on the test set (made of selected archive documents from the 19691989 era) can go up to $87 \%$. This allows us to consider the application of an unsupervised LM adaptation scheme. In our case it means that we can try to use the automatically acquired transcriptions of the target archive documents as an additional text corpus. Obviously, it will always contain a certain type and amount of errors. In order to minimize their number and ensure the correct validation in tests, we applied the following strategy:

1. The data was transcribed by the system that had been set up for the optimal performance (in the same way as in experiment $\mathrm{F}$ in Table 2.

2. The speech segments in which the transcription system identified an increased amount of noise were removed from the corpus.

3. The utterances that were classified as Slovak by the language identification module 11] were removed.

4. The documents used in the test set were removed, too.

In this way we obtained a corpus (denoted as $R A$ ) whose size was $182 \mathrm{MB}$ and that contained 26,330,195 words. Its statistics are shown in the last row of Table 1. The corpus was utilized together with all the other corpora for the LM adaptation.

\section{Experiments and Performance Evaluation}

All experiments have been done on a large test set. We randomly picked 13 complete radio news programs from the 1969-1989 period, transcribed them automatically and corrected manually. Utterances spoken in Slovak language were removed. The total duration of the audio data was 7.2 hours and the news contained 47,217 words. 
In the experiments, we focused mainly on measuring the impact of lexicons and language models on transcription accuracy and also on the Out-ofVocabulary rate. The accuracy was evaluated using the standard measure:

$$
A c c=(H-I) / N
$$

where $H$ represent the number of correctly recognized words (hits), $I$ is the number of insertions and $N$ is the total number of words in reference transcriptions.

The goal of the first experiment was to learn the baseline performance level of the system operating with the linguistic module trained for contemporary Czech (with the existing $551 \mathrm{~K}$ lexicon). The accuracy was $82.95 \%$ with the OOV rate $1.64 \%$.

In the second experiment we used the lexicon and language model built from the $R P$ corpus (i.e. the scanned and OCRed historical newspapers). In spite of a much smaller amount of training data (compared to the corpus of modern Czech) and a smaller lexicon ( $454 \mathrm{~K}$ words), the OOV rate decreased to $1.45 \%$ and the accuracy improved to $84.58 \%$. This can be explained by a very tight match between the newspaper text and the speech used in official media, which was a typical feature during the communist regime. In the next series of experiments,

Table 2. Impact of text corpora used for creating lexicons and LMs on transcription accuracy of archive news from 1969-1989 period. Experiments were performed with 1-pass and 2-pass decoding schemes. (The latter used unsupervised speaker adaptation between the two passes.)

\begin{tabular}{|c|c|c|c|c|c|}
\hline & Data used for creating lexicon and LM & $\begin{array}{c}\text { Decoding } \\
\text { scheme }\end{array}$ & \begin{tabular}{|c|} 
Lexicon \\
size
\end{tabular} & $\begin{array}{c}\text { Acc } \\
{[\%]}\end{array}$ & $\begin{array}{c}\text { OOV } \\
{[\%]}\end{array}$ \\
\hline $\mathrm{A}$ & CPaper+CBroad & 1-pass & 551,771 & 82.95 & 1.64 \\
\hline $\mathrm{B}$ & $R P$ & 1-pass & 454,074 & 84.58 & 1.45 \\
\hline $\mathrm{C}$ & RP+Parl+Retro & 1-pass & 455,031 & 84.84 & 1.34 \\
\hline $\mathrm{D}$ & CBroad + RP + Parl+Retro & 1-pass & 529,489 & 85.21 & 1.32 \\
\hline $\mathrm{E}$ & CPaper + CBroad $+R P+$ Parl + Retro & 1-pass & 559,139 & 85.67 & 1.32 \\
\hline $\mathrm{F}$ & CPaper + CBroad + RP + Parl + Retro & 2-pass & 559,139 & 87.54 & 1.32 \\
\hline $\mathrm{G}$ & CPaper + CBroad $+R P+$ Parl + Retro $+R A$ & 1-pass & 559,139 & 86.23 & 1.32 \\
\hline & CPaper + CBroad $+R P+$ Parl + Retro $+R A$ & 2-pass & \begin{tabular}{|l|}
559,139 \\
\end{tabular} & 88.19 & 1.32 \\
\hline
\end{tabular}

we were gradually increasing the size of the training data, by adding the other historical texts first and mixing them with contemporary resources later. The results are summarized in Table 2 . The best performance with $A c c=85.67 \%$ was achieved when all the available test resources were employed.

So far, all the tests were done with the LVCSR system running in a 1-pass mode. It is possible, however, to set up the speech decoding engine so that it utilizes a 2-pass approach. It means that after the standard first pass, the system tries to segment the speech signal into parts spoken by different speakers, and for each one it utilizes the recognized phoneme sequences for an unsupervised 
adaptation of the acoustic model [12. The speaker adapted model is used in the second pass. The transcription process gets slower (usually by factor 2 to 3 ), but the results are better. In our case, the accuracy increased from $85.67 \%$ to 87.54 $\%$ (see experiments $\mathrm{E}$ and $\mathrm{F}$ ).

This best performing system was used to transcribe automatically all archive documents from the 1969-1989 period and to create a domain specific text corpus (denoted as $R A$ ) described in details in section 4.3. When added to the other corpora, the accuracy reached $86.23 \%$ in the first pass and $88.19 \%$ after the second pass. At the moment, this is the best result we are able to achieve.

\section{Conclusion and Future Work}

In this paper, we present a system whose aim is to transcribe the historical archive of spoken documents collected by Czech and Czechoslovak Radio during the last 90 years. Within those 9 decades, the language has evolved substantially, mainly due to political and social changes in the society. The linguistic module of the transcription system has to follow this evolution, which means that the lexicon and language model must be adapted to each historical epoch. For contemporary speech, it was not that difficult because there exists a large amount of electronic texts representing modern written and spoken language.

For previous epochs, however, suitable and applicable language resources are hard to find. In our case, we utilized scanned and OCRed newspapers as the main source and complemented it by additional data, such as historical parliament files and subtitles from retro TV broadcasting. These resources helped us to adapt the lexicon and language model, which significantly improved the transcription accuracy. On the large test set representing radio news from 1969-1989 period, the accuracy got over $88 \%$, which is $5 \%$ better than with the original linguistic module. We have also shown that at this level it is possible to utilize the automatically transcribed archive documents for unsupervised adaptation of the language model.

As the next step, we want to pick up about 100 news documents evenly representing the 1969-1989 period. Their automatic transcriptions will be manually checked and corrected by students collaborating on the project. These corrected texts will serve several purposes:

a) to identify broadcast specific OOV words (e.g. names of news editors, words and terms typical for radio speech, etc) which will be added to the lexicon,

b) to get a large development set on which we can optimize weighting factors applied for different corpora during the language model computation, and also

c) to retrain the existing acoustic model so that it better fits audio signal recorded 30 to 40 years ago.

Furthermore, we plan to use the proposed approach also for the previous historical epochs, i.e. 1948-1968 and 1923-1947.

Acknowledgments. The research was supported by the Czech Ministry of Culture - project no. DF11P01OVV013 in program NAKI. 


\section{References}

1. Hansen, J.H.L., Huang, R., Zhou, B., Seadle, M., Deller, J.R., Gurijala, A.R., Kurimo, M., Angkititrakul, P.: SpeechFind: Advances in Spoken Document Retrieval for a National Gallery of the Spoken Word. IEEE Trans. on Speech and Audio Processing 13(5), 712-730 (2005)

2. Ordelman, R.J.F., de Jong, F.M.G., Huijbregts, M.A.H., van Leeuwen, D.A.: Robust audio indexing for Dutch spoken-word collections. In: 16th Int. Conference of the Association for History and Computing. Humanities, Computers and Cultural Heritage, Amsterdam, pp. 215-223 (2005)

3. Christel, M.G., Stevens, S.M., Maher, B.S., Richardson, J.: Enhanced exploration of oral history archives through processed video and synchronized text transcripts. In: Proceedings of the International Conference on Multimedia, pp. 1333-1342. $\operatorname{ACM}(2010)$

4. Byrne, W., et al.: Automatic recognition of spontaneous speech for access to multilingual oral history archives. IEEE Trans. Speech Audio Process. 12(4), 420-435 (2004)

5. Goldman, J., et al.: Accessing the spoken word. International Journal on Digital Libraries 5(4), 287-298 (2005)

6. Nouza, J., Blavka, K., Bohac, M., Cerva, P., Zdansky, J., Silovsky, J., Prazak, J.: Voice Technology to Enable Sophisticated Access to Historical Audio Archive of the Czech Radio. In: Grana, C., Cucchiara, R. (eds.) MM4CH 2011. CCIS, vol. 247, pp. 27-38. Springer, Heidelberg (2012)

7. Nouza, J., Blavka, K., Zdansky, J., Cerva, P., Silovsky, J., Bohac, M., Chaloupka, J., Kucharova, M., Seps, L.: Large-scale processing, indexing and search system for Czech audio-visual cultural heritage archives. In: IEEE 14th International Workshop on Multimedia Signal Processing (MMSP), pp. 337-342 (2012)

8. Nouza, J., Blavka, K., Cerva, P., Zdansky, J., Silovsky, J., Bohac, M., Prazak, J.: Making Czech Historical Radio Archive Accessible and Searchable for Wide Public. Journal of Multimedia 7(2), 159-169 (2012)

9. Czech Parliament webpages, http://www.psp.cz

10. Kneser, R., Ney, H.: Improved backing-off for m-gram language modeling. In: Proc. of IEEE Int. Conf. on Acoustics, Speech and Signal Processing, Detroit, pp. 181-184 (1995)

11. Nouza, J., Cerva, P., Silovsky, J.: Dealing with Bilingualism in Automatic Transcription of Historical Archive of Czech Radio. In: Petrosino, A., Maddalena, L., Pala, P. (eds.) ICIAP 2013 Workshops. LNCS, vol. 8158, pp. 238-246. Springer, Heidelberg (2013)

12. Cerva, P., Palecek, K., Silovsky, J., Nouza, J.: Using Unsupervised Feature-Based Speaker Adaptation for Improved Transcription of Spoken Archives. In: Proc. of Interspeech 2011, Florence, pp. 2565-2568 (2011) 